\title{
Directional reflectance studies in support of the Radiometric Calibration Test Site (RadCaTS) at Railroad Valley
}

Jeffrey S. Czapla-Myers, Craig A. Coburn, Kurtis J. Thome, Brian N. Wenny, Nikolaus J. Anderson

Jeffrey S. Czapla-Myers, Craig A. Coburn, Kurtis J. Thome, Brian N. Wenny, Nikolaus J. Anderson, "Directional reflectance studies in support of the Radiometric Calibration Test Site (RadCaTS) at Railroad Valley," Proc. SPIE 10764, Earth Observing Systems XXIII, $107640 Z$ (7 September 2018); doi: 10.1117/12.2320756

EPIE Event: SPIE Optical Engineering + Applications, 2018, San Diego, California, United States 


\title{
Directional reflectance studies in support of the Radiometric Calibration Test Site (RadCaTS) at Railroad Valley
}

\author{
Jeffrey S. Czapla-Myers ${ }^{\mathrm{a}}$, Craig A. Coburn ${ }^{\mathrm{b}}$, Kurtis J. Thome ${ }^{\mathrm{c}}$, \\ Brian N. Wenny ${ }^{\mathrm{d}}$, and Nikolaus J. Anderson ${ }^{\mathrm{a}}$ \\ ${ }^{\mathrm{a} C}$ College of Optical Sciences, University of Arizona, 1630 E University Blvd, Tucson, AZ 85721-0094, USA \\ ${ }^{b}$ Dept of Geography, University of Lethbridge, 4401 University Dr, Lethbridge, AB T1K 3M4, Canada \\ 'NASA Goddard Space Flight Center, Code 618, Greenbelt, MD 20771-0001, USA \\ ${ }^{\mathrm{d}}$ Science Systems and Applications Inc., 10210 Greenbelt Road, Suite 600, Lanham, MD 20706-6329, USA
}

\begin{abstract}
The Radiometric Calibration Test Site (RadCaTS) is a suite of commercial and custom instruments used to make measurements of the surface reflectance and atmosphere throughout the day at Railroad Valley, Nevada. It was developed in response to the need for daily radiometric calibration data for the vast array of Earth-observing sensors on orbit, which is continuously increasing as more nations and private companies launch individual environmental satellites as well as large constellations. The current suite of instruments at RadCaTS includes five ground-viewing radiometers (GVRs), four of which view the surface in a nadir-viewing configuration. Many sensors such as those on Landsat- 7 and Landsat- 8 view Railroad Valley within $3^{\circ}$ of nadir, while others such as those on Sentinel-2A and -2B, RapidEye, Aqua, Suomi NPP, and Terra can view Railroad Valley at off-nadir angles. Past efforts have shown that the surface bidirectional reflectance distribution function (BRDF) has minimal impact on vicarious calibration uncertainties for views $<10^{\circ}$, but the desire to use larger view angles has prompted the effort to develop a BRDF correction for data from RadCaTS. The current work investigates the application of Railroad Valley BRDF data derived from a BRF camera developed at the University of Arizona in the 1990s (but is no longer in use) to the current RadCaTS surface reflectance measurements. Also investigated are early results from directional reflectance studies using a mobile spectro-goniometer system during a round-robin field campaign in 2018. This work describes the preliminary results, the effects on current measurements, and the approach for future measurements.
\end{abstract}

Keywords: radiometric calibration, surface reflectance, BRDF, BRF, goniometer, RadCaTS, RadCalNet

\section{INTRODUCTION}

Ground-based radiometric calibration techniques are routinely used to monitor changes to satellite sensors, and to validate the algorithms used to produce surface reflectance products. ${ }^{1-12}$ The Remote Sensing Group (RSG) of the College of Optical Sciences at the University of Arizona has provided preflight and post-launch vicarious radiometric calibration data to national and commercial satellite operators for $25^{+}$years. ${ }^{13,14}$ Post-launch radiometric calibration techniques include in situ measurements at suitable test sites, which include Railroad Valley, Nevada, USA. The reflectance-based approach has typically been used by many groups involved in radiometric calibration, and it requires making surface reflectance and atmospheric measurements for a given time of interest. ${ }^{15}$ The atmospheric and surface reflectance measurements are used as input into a radiative transfer code to determine the top-of-atmosphere (TOA) quantity of interest. TOA results are then band averaged to the sensor under test for direct comparison with the sensor imagery.

The reflectance-based approach to vicarious radiometric calibration requires that personnel be present on site during the satellite overpass time of interest. This procedure has worked well in the past, when there was generally a smaller number of flagship satellites to be calibrated. An increase in government and commercial satellite launches over the past decade has made it more financially and logistically challenging to collect in situ data using on-site personnel. RSG originally developed RadCaTS to collect suitable ground and atmospheric data in addition to those collected with ground personnel. Studies were conducted to determine the amount and location of GVRs that would be required to spatially sample the $1-\mathrm{km}^{2}$ area that defines RadCaTS, ${ }^{16,17}$ as well as the amount of temporal data that needs to be collected throughout the year to maintain a certain level of accuracy for radiometric calibration trending. ${ }^{18}$ Past results show that previously-ignored

Earth Observing Systems XXIII, edited by James J. Butler, Xiaoxiong (Jack) Xiong, Xingfa Gu, Proc. of SPIE Vol. 10764, 107640Z · @ 2018 SPIE · CCC code: 0277-786X/18/\$18 · doi: 10.1117/12.2320756 
effects are becoming more important as the precision and accuracy of the reflectance-based approach have improved..$^{12,19}$ One of these effects is due to the bidirectional nature of the surface reflectance. Early studies of RSG test sites indicated little or no impact from directional reflectance effects of the test sites commonly used by RSG for sensor view angles out to as much as $30^{\circ} .{ }^{20}$ Modeling studies showed that ignoring surface directional reflectance effects on the diffusely-scattered light would cause little error in the predicted at-sensor radiance for the group's test sites for view angles as extreme as $45^{\circ} .{ }^{21}$ Past studies ignored the case of sensor view angles exceeding $10^{\circ}$ while the sensor azimuth is within $60^{\circ}$ of the solar azimuth angle. Such geometries are now becoming more commonplace with the development of more agile and pointable narrow-swath sensors, as well as the result of the automated field collections providing additional data sets with these varying geometries. For example, attempts to calibrate the backward-looking telescope of ASTER using nadir-looking measurements of reflectance led to errors as large as $20 \%$ for winter dates. ${ }^{22}$

RadCaTS has been using the current GVRs since 2011, so a reasonable amount of high-quality data have been captured for sensors that view Railroad Valley at various angles. Sensors such as Landsat-7 ETM+ and Landsat-8 OLI view Railroad Valley on a 16-day repeat cycle, but other sensors such as Terra and Aqua MODIS, and Sentinel-2A and -2B MSI view Railroad Valley at multiple angles. There are also sensors such as ASTER and the DigitalGlobe WorldView series that have the capability to point off-nadir. In addition, many of the sensors used in the RadCalNet beta testing phase, such as RapidEye, view Railroad Valley at various non-nadir view angles. In order to provide the highest quality data to RadCaTS and RadCalNet users, directional reflectance studies are underway using a variety of techniques including the results of in situ measurements made during a round robin field campaign in May 2018, and prior measurements using an RSG BRF camera. The results from these techniques will be applied to RadCaTS and RadCalNet results from 2012 and 2014, respectively, to present.

\section{METHODOLOGY}

\subsection{RadCaTS}

RadCaTS was originally developed as a proof-ofconcept method using inexpensive GVRs based on LEDs for detectors ${ }^{23}$ but in 2011-2012 more advanced GVRs that addressed the shortcomings of LEDs were developed, tested, and deployed to RadCaTS (Fig. 1). ${ }^{24,25}$ The current 8-channel design uses a thermoelectric cooler to maintain a constant focal plane temperature throughout the day. The spectral selection is controlled using interference filters, each of which is measured in RSG's laboratory. Each GVR is a standalone unit that is solar powered and connected to a central Wi-Fi station that also contains a satellite uplink to the University of Arizona. There are currently five GVRs deployed to RadCaTS: four in a nadirviewing configuration, and one in the same viewing configuration as GOES-16 (GOES-East). Two additional GVRs will be added to RadCaTS in 20182019: one in a nadir-viewing configuration, and one in the GOES-17 (GOES-West) viewing configuration. Atmospheric measurements are made using Cimel sun photometers that have been operating at Railroad

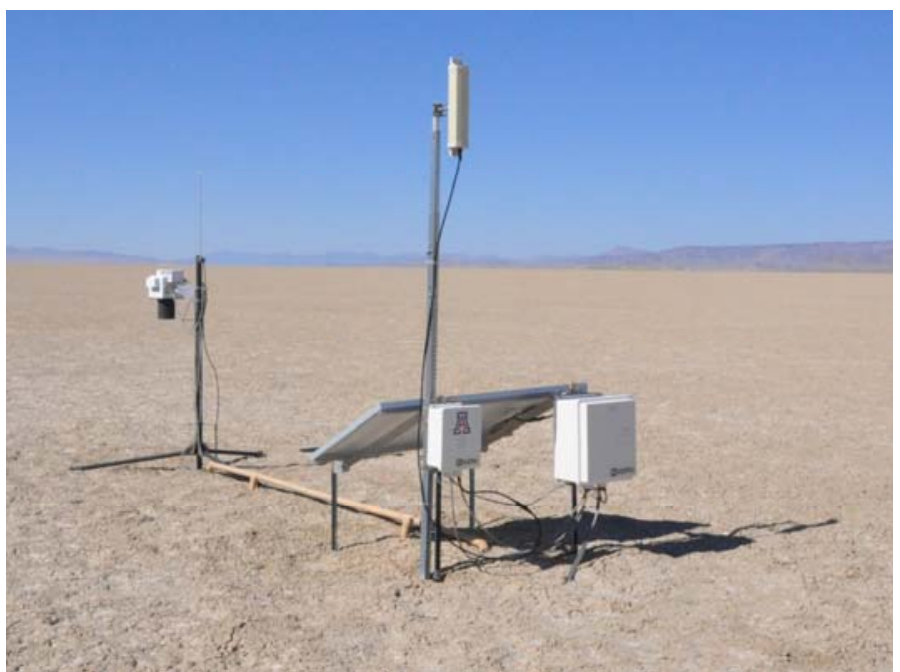

Fig. 1. GVR at Railroad Valley, including the solar panel for charging, electronics boxes, and Wi-Fi antenna. The focal plane of the GVR is $1.5 \mathrm{~m}$ above the ground.

Valley since 2001. The measurement routine follows the typical AERONET measurement protocol. ${ }^{26} \mathrm{RSG}$ currently operates three Cimel units $(15,314$, and 786$)$, which are routinely exchanged for periodic calibration at NASA GSFC.

The multispectral GVR measurements of surface reflectance in each of the eight bands are averaged for a given date and time of interest. They are converted to a hyperspectral surface reflectance $(400-2500 \mathrm{~nm})$ by determining the best fit to a library of hyperspectral measurements made by RSG using a portable spectroradiometer from 2000-2018. The final hyperspectral surface reflectance is used in the MODTRAN radiative transfer code with an exoatmospheric solar irradiance to determine a top-of-atmosphere spectral radiance (or reflectance). The exoatmospheric irradiance is specific to each satellite sensor under test, but in the case of RadCalNet, the Thuillier model is used since it is recommended by CEOS. ${ }^{27}$ 

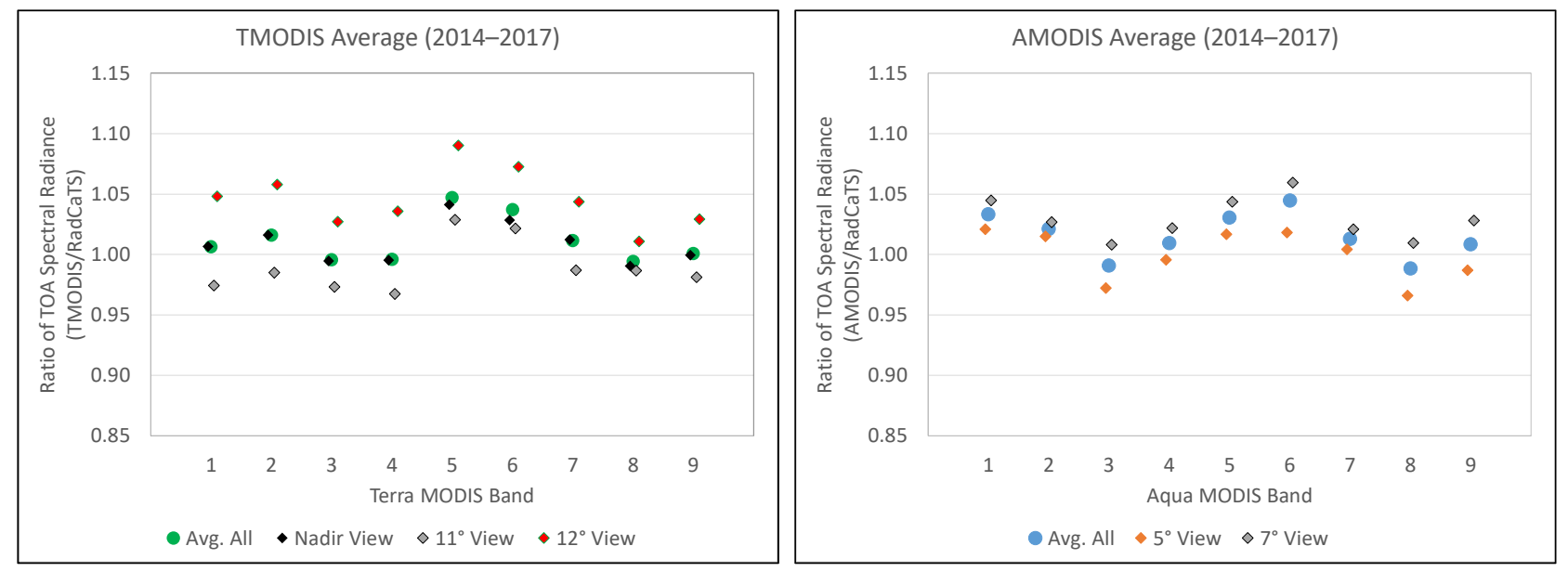

Fig. 2. Recent results for Terra (left) and Aqua (right) MODIS at RadCaTS, separated into the average, and also the nadir and nearnadir view angles. The uncertainty of the measurements is approximately $\pm 4 \%$, but the uncertainty bars are omitted for clarity.

Fig. 2 shows an example of recent results for Terra and Aqua MODIS, which view Railroad Valley at three (Terra) and two (Aqua) view angles $<15^{\circ}$, summarized in Table 1. When the results in Fig. 2 are grouped into separate view angles, the effects of view geometry and surface BRDF are clearly observed for the data collected from 2014-2017. The results from these and other sensors have expedited the current effort to characterize the BRDF of Railroad Valley. Though, it should be pointed out that the differences observed between the multiple view angles of MODIS at Railroad Valley lead to noticeable differences in all bands, none of the differences are statistically significant, and all of the differences are within the absolute uncertainty of the RadCaTS results.

\subsection{Bidirectional Reflectance Factor Camera}

RSG developed an imaging system for the groundbased characterization of test site directional reflectance based on a scientific-grade CCD array coupled to a fisheye lens. ${ }^{20,28,29}$ The imaging array is a $1024 \times 1024$-pixel silicon CCD array that is coupled to a Nikkor 8-mm fisheye lens, which provides a $180^{\circ}$ full field of view (Fig. 3). Spectral selection is controlled using four interferences filters with center wavelengths at $470,575,660$, and $835 \mathrm{~nm}$. One of the main advantages of using an imaging system to measure directional reflectance is that the entire hemisphere can be captured in a single image. The tradeoff is multispectral information versus the hyperspectral information that can be captured using a nonimaging portable spectroradiometer. The issue with decreased spectral information can be reduced by using suitable sites that have slowly varying spectral characteristics. The BRF camera is not currently in use, mainly due to
Table 1. Terra and Aqua MODIS overpass time, view zenith angle (VZA), and view azimuth angle (VAA) for near-nadir overpasses at Railroad Valley. The repeat time for each view angle is 16 days.

\begin{tabular}{|ccc|}
\hline $\begin{array}{c}\text { Terra } \\
\text { Time (UTC) }\end{array}$ & VZA (degrees) & VAA (degrees) \\
\hline $18: 33$ & 12.0 & 102.5 \\
$18: 39$ & 0.3 & 104.4 \\
$18: 46$ & 11.6 & 284.0 \\
\hline
\end{tabular}

\begin{tabular}{|ccc|}
\hline $\begin{array}{c}\text { Aqua } \\
\text { Time (UTC) }\end{array}$ & VZA (degrees) & VAA (degrees) \\
\hline $20: 48$ & 4.8 & 256.9 \\
$20: 54$ & 7.0 & 75.9 \\
\hline
\end{tabular}

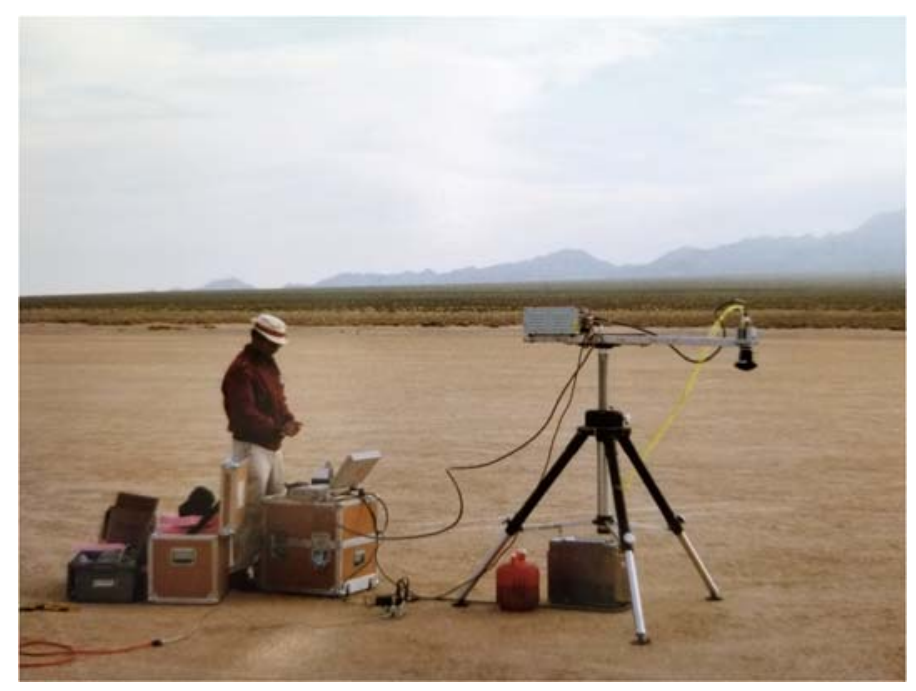

Fig. 3. BRF camera in use at Ivanpah Playa, California. 
the complex cooling system required to maintain the $\mathrm{CCD}$ array at $-32^{\circ} \mathrm{C} \pm 1^{\circ} \mathrm{C}$. The heat from the thermoelectric cooler is dissipated through an ethylene-glycol cooling system coupled to an ice-water bath, which is cumbersome to deploy in the field. In situ field measurements were made between 1997-2000 at Railroad Valley, Nevada, Ivanpah Playa, California, and White Sands Missile Range, New Mexico. In the study presented here, the historical results of the Railroad Valley measurements previously made with the BRF camera are used to scale the current surface reflectance values determined using the nadir-viewing GVRs at RadCaTS.

\subsection{University of Lethbridge Goniometer System}

While not part of the previous instrument installation, the growing need for a detailed diurnal characterization of the Railroad Valley BRDF resulted in the deployment of a goniometer system with the ability to provide the sampling of the surface BRDF needed for this project. The BRDF is the intrinsic property of a surface that describes the angular distribution of radiation reflected by the surface for all angles of exitance and under any given illumination geometry. ${ }^{30}$ The BRDF of a surface is, by definition the summation of infinitesimally small angles and, therefore, all direct measurements are samples from the BRDF, $\rho$ :

$$
\rho=\frac{d L_{r}\left(\theta_{i}, \phi_{i}, \theta_{r}, \phi_{r} \lambda\right)}{d E_{i}\left(\theta_{i}, \phi_{i}, \lambda\right)}\left[s r^{-1}\right]
$$

where $d L\left[\mathrm{~W} \mathrm{~m}^{-2} \mathrm{sr}^{-1}\right]$ is the differential radiance reflected into the differential solid angle, $d E$ [W $\left.\mathrm{m}^{-2}\right]$ is the differential incident irradiance for a given zenith angle $(\theta)$ and azimuth angle $(\phi)$, for any wavelength $(\lambda)$. Subscripts $r$ and $i$ indicate reflected and incident quantities, respectively.

In practice, the illumination irradiance is provided not only from the Sun but from the sky that is visible from the target (hemispherical in most instances) and the size of the observing field of view (FOV) is normally conical in nature. The quantity rendered is therefore a hemispherical-conical reflectance factor (HCRF).

The instrument deployed in May 2018 was the University of Lethbridge Goniometer System 2 (ULGS-2). ${ }^{31}$ The ULGS-2 instrument is a robotic spectro-goniometer system that has a quarter circle arc mounted to a beam that serves as a pivot for the arc (Fig. 4). This allows for a complete hemispherical sampling of the surface as the spectrometer is positioned along the arc and the arc is rotated about pivot. The instrument has a large operating radius of $2 \mathrm{~m}$. Two Ocean Optics USB-4000 spectrometers are used for measuring the target and monitoring downwelling illumination conditions. The target observing spectrometer uses an $8^{\circ}$ foreoptic, produces a ground sampling pattern that is $\sim 60 \mathrm{~cm}$ at the instrument focal plane, while the downwelling instrument incorporates a cosine receptor made from Spectralon.

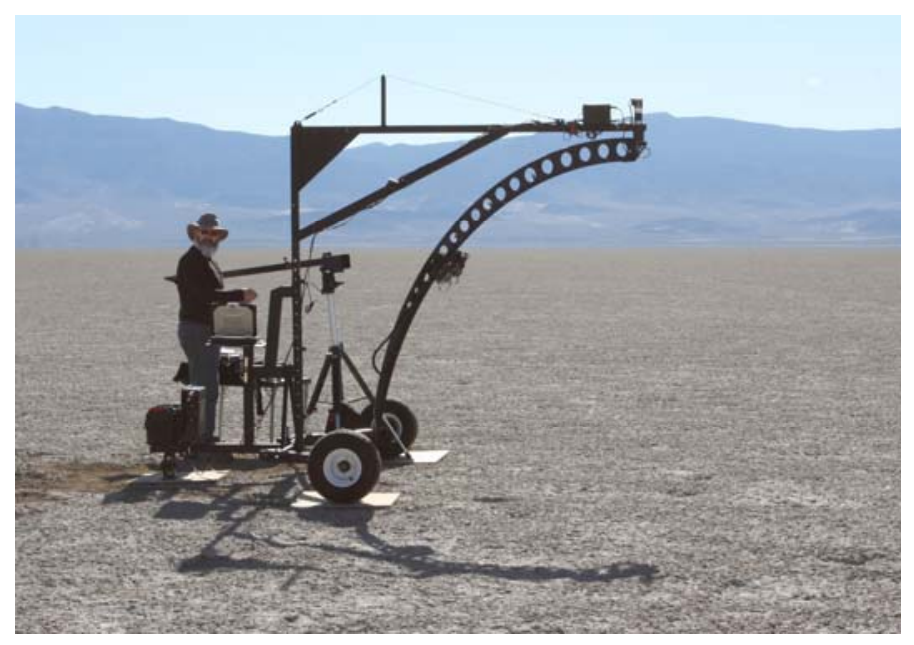

Fig. 4. ULGS-2 in operation at Railroad Valley.

The ULGS-2 is a fully transportable field goniometer system, and with the quarter arc design, has a broader sampling range than is typical of single-target goniometer systems. It also incorporates a real-time spectral reflectance calculation by using the data collected from the downwelling spectrometer to compute spectral reflectance as a ratio of the downwelling signal to the upwelling signal from a Spectralon panel. An Analytical Spectral Devices (ASD) FieldSpec Full-Range Spectroradiometer was used to gather spectra of the Railroad Valley surface target at the end of each measurement cycle to provide calibration data for the spectrometers mounted on the ULGS-2. 


\section{DATA}

\subsection{RadCaTS Data and BRDF Correction Using Historical BRF Camera Data}

RadCaTS data are collected throughout the day on a daily basis. Each of the GVRs make measurements every two minutes when a threshold voltage is reached in the $850-\mathrm{nm}$ channel. This is performed to save power by turning the thermoelectric cooler off when cloudy conditions exist, and also at night. The Cimel photometer takes data by following the AERONET protocol, and the data are uploaded to NASA GSFC for daily processing and online distribution. The AERONET collection times are based on air mass and typically not equally spaced throughout the day, so the values for Angstrom exponent, aerosol optical depth at $500 \mathrm{~nm}$, and water vapor, are interpolated between the two nearest points in time for a given time of interest.

The surface reflectance is determined by comparing the downwelling solar irradiance to the reflected radiance measured by the GVRs, with the atmospheric transmittance being determined through the use of MODTRAN with AERONET data used as input. The individual GVR measurements are averaged for each of the eight bands to create a multispectral surface reflectance. These data are then fit to one of $\sim 700$ hyperspectral reference data sets that were collected by RSG using portable hyperspectral spectroradiometers from 2000-2018. The final hyperspectral surface reflectance $(400-2500 \mathrm{~nm})$ is used once again in MODTRAN to determine the TOA spectral radiance (or reflectance) for the sensor under test. In the case of RadCalNet, the surface reflectance data are downsampled to 10-nm intervals and uploaded to NASA GSFC for final propagation to TOA reflectance. The final RadCalNet product is the TOA reflectance (400-2500 nm) measured every $10 \mathrm{~nm}$, every 30 minutes, from 09:00-15:00 local standard time (17:00-23:00 UTC at Railroad Valley). The RadCaTS data presented in this study begin with the standard methodology used to determine the nadir-viewing surface reflectance and TOA spectral radiance (or reflectance) described in Section 2.1.

An example of the RadCaTS-derived surface reflectance for one of the round-robin field dates is shown in Fig. 5 for GVR band $3(500 \mathrm{~nm})$ on 3 May 2018. The negative solar zenith angles (SZAs) correspond to morning, while the positive SZAs correspond to afternoon. In order to correct the nadirviewing RadCaTS data, the historical BRF camera data (Section 2.2) are used to create a surface reflectance correction based on the solar and view geometry configuration. The newly-scaled hyperspectral surface reflectance is then used as the input into MODTRAN to determine the final TOA spectral radiance (or reflectance).

Five RadCaTS data sets have been used in this study: one for each off-nadir view configuration of Terra and Aqua MODIS, and one for the Terra MODIS overpass that occurred during the round-robin field campaign on 3 May 2018. In each of these cases, the surface reflectance determined using the nadir-viewing GVRs has a correction factor applied based on the earlier

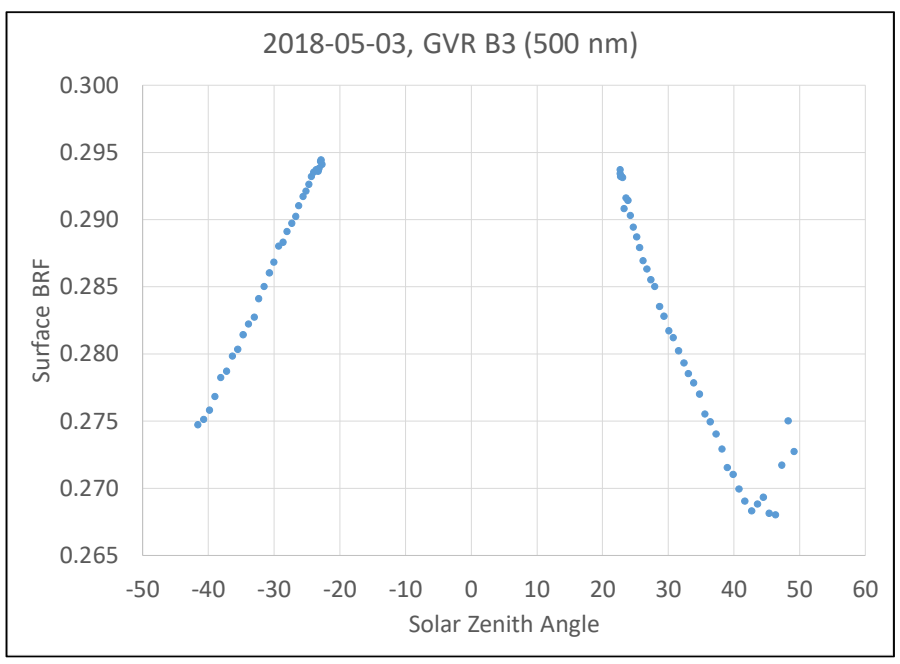

Fig. 5. An example of the Railroad Valley BRF at $500 \mathrm{~nm}$ (GVR band 3) as a function of solar zenith angle, measured on 3 May 2018 with RadCaTS nadir-viewing GVRs.

results from the BRF camera described in Section 2.2. It should be noted that the BRDF correction in this initial study is only applied to the VNIR spectral region since the four BRF camera wavelengths range from $470-835 \mathrm{~nm}$.

\subsection{ULGS-2 Data}

During the round-robin field campaign in May 2018, the ULGS-2 sampled the Railroad Valley surface every 30 minutes near the center of the $1-\mathrm{km}^{2}$ region of interest that defines RadCaTS. This corresponds to approximately $5^{\circ}$ changes in the solar zenith angle over a range of solar angles typifying the surface condition for the majority of Earth Observing (EO) satellite observation angles. The ULGS- 2 was programmed to sample the BRDF at $10^{\circ}$ increments in both zenith and azimuth with the zenith restricted to a maximum of $60^{\circ}$.

The data presented here are from 3 May 2018. The sky conditions were generally favorable during the early morning into the midafternoon period. Fig. 6 shows the variation in solar elevation over the measurement cycle. Measurements were 
stopped in the early afternoon due to the presence of clouds. The relatively uniform nature of the surface, however, should show similar reflectance anisotropy in the afternoon period as the solar zenith angles are repeated. The only variable would be the alteration of the solar azimuth relative to the instrument position.

Fig. 7 shows the variation HCRF for a single wavelength $(580 \mathrm{~nm})$ from the morning, solar noon, and afternoon period. The pattern of the polar plot shows the relative symmetry of these measurements over time. The polar plots are oriented in the solar principle plane vertical with the backscatter direction towards the bottom of the figure. The general pattern shows a weakening backscatter as the solar angles increase in zenith. At solar noon, the surface is quite diffuse. The regional "hot spot" observed in the forward scatter direction (at approximately $200^{\circ}$ ) is

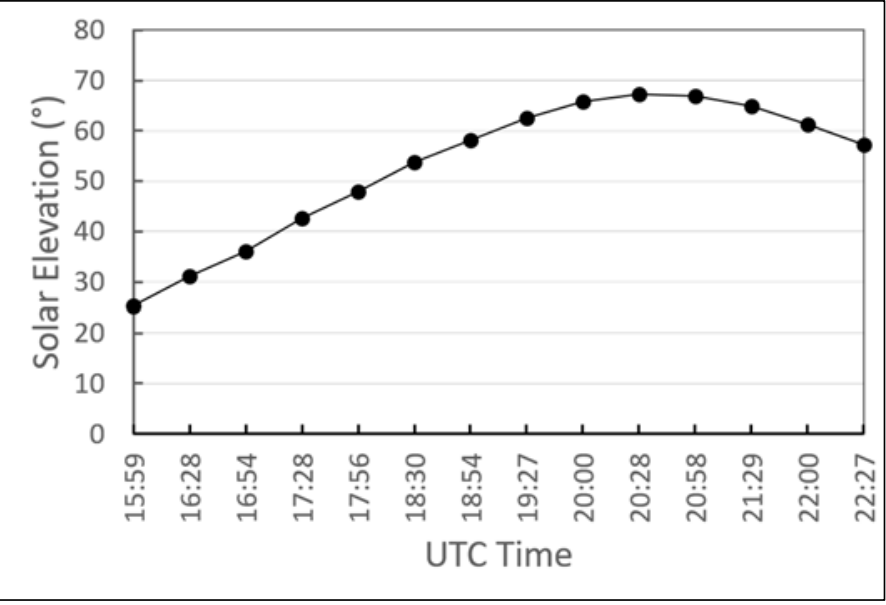

Fig. 6. Solar elevation angle change over measurement cycle. most likely sun glint caused by the quartz grains located in the depressions of the surface. This effect is still being investigated. The strength of the reflectance anisotropy also varies per wavelength. Fig. 8 shows five different wavelengths for 22:00 UTC. The variation at shorter wavelengths is lower, as the surface reflectance is also lower at these wavelengths. Longer wavelengths show the greatest degree of variation.

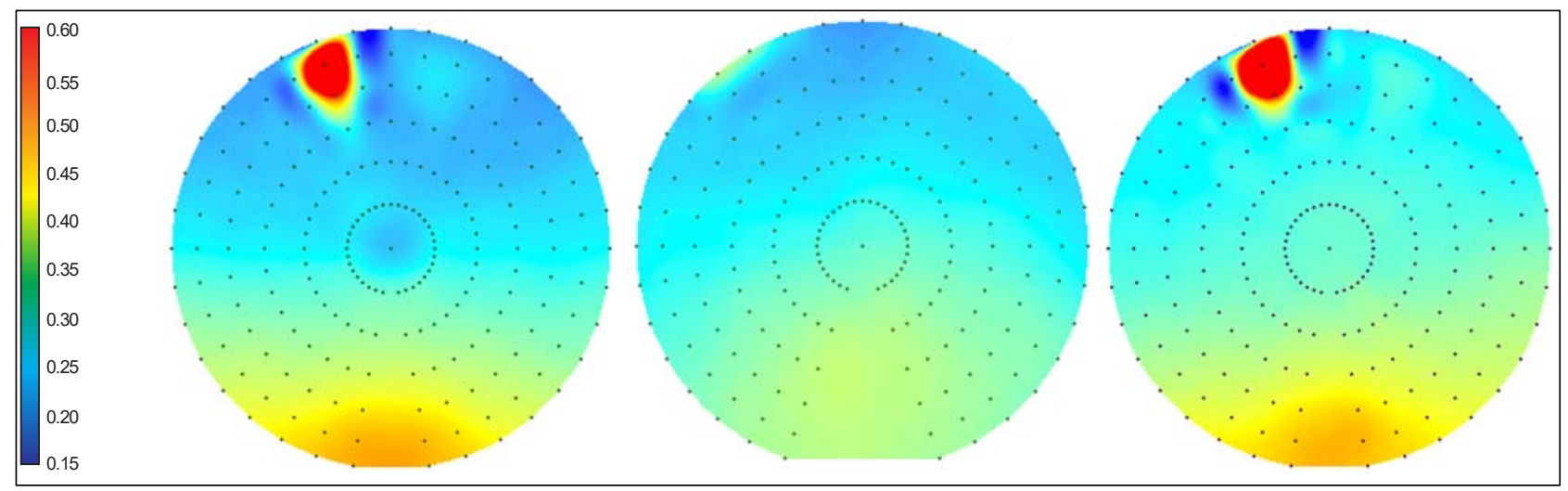

Fig. 7. HCRF plots determined using ULGS-2 from morning (18:30 UTC), solar noon (20:58 UTC), and afternoon (22:27 UTC). The general trend is towards a more diffuse reflectance variation at solar noon with increasing anisotropy that appears to be symmetrical. The figures are oriented with the solar principal plane in the vertical direction with the backscatter direction towards the bottom of the figure. The "hot spot" observed in the morning and afternoon periods is thought to be caused by Sun glint from the quartz grains present on the surface. Each ring of dots corresponds to a view zenith angle increase of $10^{\circ}$ (e.g. $0^{\circ}-60^{\circ}$ for the plots shown here).

\section{RESULTS}

The results of RadCaTS measurements during two overpasses of Terra and Aqua MODIS are shown in Fig. 9 and Fig. 10. Each of the two cases represent one view azimuth angle (VAA) from the east (VAA $<180^{\circ}$ ) and one VAA from the west $\left(\mathrm{VAA}>180^{\circ}\right.$ ). In each case, the RadCaTS-derived surface BRF has been adjusted with historical results obtained using the BRF camera at Railroad Valley. As previously mentioned, the results for this study are restricted to the MODIS VNIR bands (1-4, 8, and 9). Fig. 9 shows the results for two off-nadir view geometries of Terra MODIS, and Fig. 10 shows the results for two off-nadir view geometries for Aqua MODIS. In both cases, the BRDF-corrected RadCaTS results generally show better agreement with MODIS. The data from ULGS-2 are currently being processed, but initial results indicate that the BRDF correction is comparable to those determined using the historical BRF camera data. 


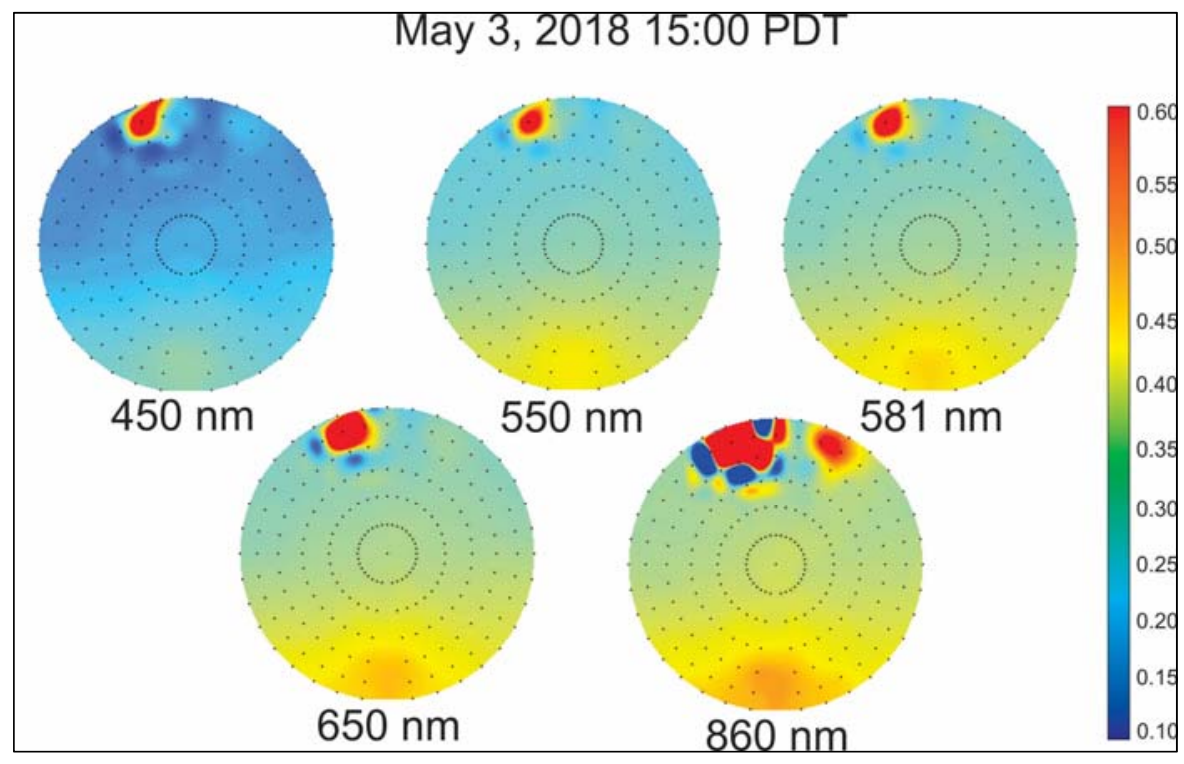

Fig. 8. Reflectance anisotropy with wavelength, measured by the ULGS-2 instrument. The pattern of anisotropic behavior is stronger at longer wavelengths. Each ring of dots corresponds to a view zenith angle increase of $10^{\circ}$ (e.g. $0^{\circ}-60^{\circ}$ for the plots shown here).
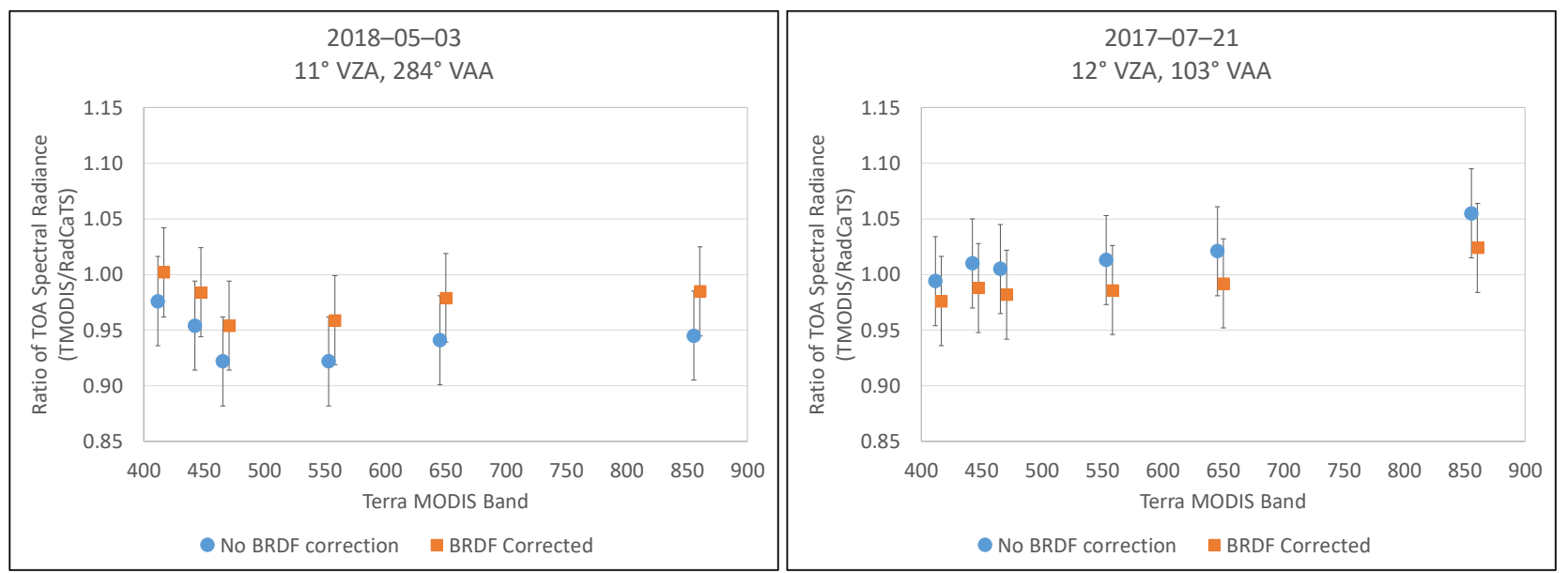

Fig. 9. BRDF-corrected RadCaTS results for two Terra MODIS overpasses, viewing from the west (left) and from the east (right). The VNIR MODIS bands shown here are 1-4, 8, and 9. VZA and VAA are the view zenith angle, and the view azimuth angle, respectively.

\section{SUMMARY}

The current RadCaTS configuration has been in its current form since 2012, and surface reflectance and atmospheric data have been provided to RadCalNet from 2014 to present. The RadCalNet web portal was opened to all registered users in Jul 2018, and it is anticipated that the number of users and sites will continue to increase with time. This work revisits previous RSG directional reflectance studies that used a four-channel BRF camera, while also incorporating new measurements completed during a round robin field campaign in May 2018. The instruments included the nadir-viewing RadCaTS instruments as well as the ULGS-2 spectrogoniometric system, which is specifically designed to measure the hyperspectral HCRF of ground surfaces. The results of the round-robin field measurements are currently being analyzed, and a final correction will be determined for future use in RadCaTS. 

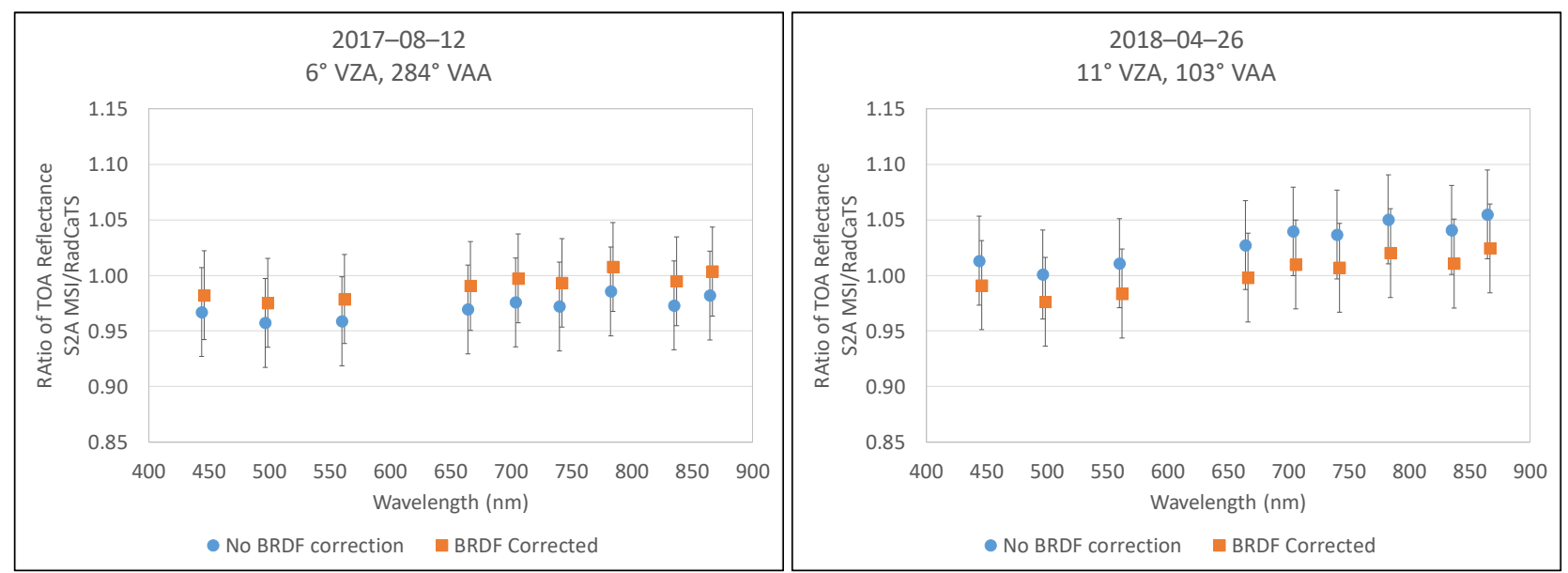

Fig. 10. BRDF-corrected RadCaTS results for two Aqua MODIS overpasses, viewing from the west (left) and from the east (right). The VNIR MODIS bands shown here are 1-4, 8, and 9. VZA and VAA are the view zenith angle, and the view azimuth angle, respectively.

The agreement between RadCaTS and two Terra and two Aqua MODIS overpasses was generally improved after applying the BRDF correction that was determined using previous results from the BRF camera data. The correction was typically on the order of $2-3 \%$, and it was only applied to the VNIR bands of each sensor since the BRF camera did not collect data in the SWIR spectral region. The limited amount of RadCaTS comparisons in this study was primarily due to the manual processing of data. Once the results from ULGS-2 and the BRF camera have been compiled, a final correction will be included in the processing code and applied to RadCaTS data based on the solar and view geometries.

\section{ACKNOWLEDGEMENTS}

The authors would like to thank the Bureau of Land Management (BLM) Tonopah, Nevada, office for their assistance and permission in using Railroad Valley, and also AERONET for processing the Cimel data. The authors would also like to acknowledge NASA for funding this work.

\section{REFERENCES}

[1] S. F. Biggar, M. Dinguirard, D. I. Gellman, P. Henry, R. D. Jackson, M. S. Moran, and P. N. Slater, "Radiometric calibration of SPOT 2 HRV - A comparison of three methods." Proc. SPIE 1493, 155-162 (1991).

[2] K. J. Thome, D. I. Gellman, R. J. Parada, S. F. Biggar, P. N. Slater, and M. S. Moran, "In-flight radiometric calibration of Landsat-5 Thematic Mapper from 1984 to present." Proc. SPIE 1938, 126-130 (1993).

[3] P. N. Slater, S. F. Biggar, K. J. Thome, D. I. Gellman, and P. R. Spyak, "Vicarious Radiometric Calibrations of EOS Sensors," Journal of Atmospheric and Oceanic Technology, 13(2), 349-359 (1996).

[4] K. P. Scott, K. J. Thome, and M. R. Brownlee, "Evaluation of the Railroad Valley Playa for use in vicarious calibration." Proc. SPIE 2818, 158-166 (1996).

[5] K. J. Thome, B. G. Crowther, and S. F. Biggar, "Reflectance- and Irradiance-based Calibration of Landsat 5 Thematic Mapper," Canadian Journal of Remote Sensing, 23(4), 309-317 (1997).

[6] K. J. Thome, "Absolute radiometric calibration of Landsat 7 ETM+ using the reflectance-based method," Remote Sensing Environment, 78, 27-38 (2001).

[7] S. F. Biggar, K. J. Thome, and W. Wisniewski, "Vicarious Radiometric Calibration of EO-1 Sensors by Reference to High-Reflectance Ground Targets,” IEEE Transactions on Geoscience and Remote Sensing, 41(6), 1174-1179 (2003).

[8] K. J. Thome, S. F. Biggar, and H. J. Choi, "Vicarious calibration of Terra ASTER, MISR, and MODIS." Proc. SPIE 5542, 290-299 (2004). 
[9] B. L. Markham, M. O. Haque, J. A. Barsi, E. Micijevic, D. L. Helder, K. J. Thome, D. Aaron, and J. S. CzaplaMyers, "Landsat-7 ETM+: 12 Years On-Orbit Reflective-Band Radiometric Performance," Geoscience and Remote Sensing, IEEE Transactions on, 50(5), 2056-2062 (2012).

[10] J. S. Czapla-Myers, N. J. Anderson, and S. F. Biggar, "Early ground-based vicarious calibration results for Landsat 8 OLI." Proc. SPIE 8866, 88660S-88660S-10 (2013).

[11] J. Czapla-Myers, L. Ong, K. Thome, and J. McCorkel, "Validation of EO-1 Hyperion and Advanced Land Imager Using the Radiometric Calibration Test Site at Railroad Valley, Nevada," Selected Topics in Applied Earth Observations and Remote Sensing, IEEE Journal of, PP(99), 1-11 (2015).

[12] J. Czapla-Myers, J. McCorkel, N. Anderson, K. Thome, S. Biggar, D. Helder, D. Aaron, L. Leigh, and N. Mishra, "The Ground-Based Absolute Radiometric Calibration of Landsat 8 OLI," Remote Sensing, 7(1), 600-626 (2015).

[13] K. J. Thome, S. F. Biggar, N. Anderson, J. Czapla-Myers, R. B. Lockwood, S. J. Miller, T. W. Cooley, T. G. Chrien, S. J. Schiller, J. F. Silny, and M. A. Glennon, "Preflight and Vicarious Calibration of ARTEMIS." Proc. IEEE Geoscience and Remote Sensing Symposium (IGARSS) 1, I-249 - I-252 (2008).

[14] R. A. Barnes, J. R. E. Eplee, S. F. Biggar, K. J. Thome, E. F. Zalewski, P. N. Slater, and A. W. Holmes, "SeaWiFS transfer-to-orbit experiment," Appl. Opt., 39(30), 5620-5631 (2000).

[15] A. R. Ehsani, J. A. Reagan, and W. H. Erxleben, "Design and Performance Analysis of an Automated 10-Channel Solar Radiometer Instrument," Journal of Atmospheric and Oceanic Technology, 15(3), 697-707 (1998).

[16] J. S. Czapla-Myers, K. J. Thome, and J. H. Buchanan, "Implication of spatial uniformity on vicarious calibration using automated test sites." Proc. SPIE 6677, 66770U-10 (2007).

[17] J. S. Czapla-Myers, K. J. Thome, B. R. Cocilovo, J. T. McCorkel, and J. H. Buchanan, "Temporal, spectral, and spatial study of the automated vicarious calibration test site at Railroad Valley, Nevada." Proc. SPIE 7081, 70810I-9 (2008).

[18] K. Thome, "Sampling and uncertainty issues in trending reflectance-based vicarious calibration results." Proc. SPIE 5882, 588216-11 (2005).

[19] K. Thome, C. Cattrall, J. D'Amico, and J. Geis, "Ground-reference calibration results for Landsat-7 ETM+." Proc. SPIE 5882, 58820B-12 (2005).

[20] P. Nandy, K. J. Thome, and S. F. Biggar, "Characterization and field use of a CCD camera system for retrieval of bidirectional reflectance distribution function," Journal of Geophysical Research, 106(D11), 11957-11966 (2001).

[21] K. J. Thome, and P. Nandy, "Accuracy of ground-reference calibration of imaging spectroradiometers at large sensor view angles." Proc. SPIE 4132, 260-268 (2000).

[22] K. Thome, "Vicarious calibration of ASTER backward-looking telescope." Proc. 6296, 629611-629611-10 (2006).

[23] J. S. Czapla-Myers, K. J. Thome, and S. F. Biggar, "Design, calibration, and characterization of a field radiometer using light-emitting diodes as detectors," Applied Optics, 47(36), 6753-62 (2008).

[24] N. J. Anderson, and J. S. Czapla-Myers, "Ground viewing radiometer characterization, implementation and calibration applications: a summary after two years of field deployment." Proc. SPIE 8866, 88660N-88660N-10 (2013).

[25] N. Anderson, J. Czapla-Myers, N. Leisso, S. Biggar, C. Burkhart, R. Kingston, and K. Thome, "Design and calibration of field deployable ground-viewing radiometers," Appl. Opt., 52(2), 231-240 (2013).

[26] B. N. Holben, T. F. Eck, I. Slutsker, D. Tanré, J. P. Buis, A. Setzer, E. Vermote, J. A. Reagan, Y. J. Kaufman, T. Nakajima, F. Lavenu, I. Janjowiak, and A. Smirnov, "AERONET - A Federated Instrument Network and Data Archive for Aerosol Characterization," Remote Sensing Environment, 66, 1-16 (1998).

[27] G. Thuillier, M. Hersé, D. Labs, T. Foujols, W. Peetermans, D. Gillotay, P. C. Simon, and H. Mandel, "The Solar Spectral Irradiance from 200 to $2400 \mathrm{~nm}$ as Measured by the SOLSPEC Spectrometer from the Atlas and Eureca Missions," Solar Physics, 214(1), 1-22 (2003).

[28] P. Nandy, K. J. Thome, and S. F. Biggar, "Sensitivity analysis of a CCD-based camera system for the retrieval of bidirectional reflectance distribution function for vicarious calibration." Proc. SPIE 4132, 279-289 (2000).

[29] P. Nandy, K. J. Thome, and S. F. Biggar, "Laboratory Characterization of a CCD camera system for retrieval of bidirectional reflectance distribution function." Proc. SPIE 3870 (1999).

[30] F. E. Nicodemus, J. C. Richmond, J. J. Hsia, I. W. Ginsberg, and T. Limperis, Geometrical Considerations and Nomenclature for Reflectance, U.S. Dept. of Commerce, National Bureau of Standards, Washington (1977).

[31] C. A. Coburn, and S. D. Noble, "ULGS II: A High-Performance Field and Laboratory Spectrogoniometer for Measuring Hyperspectral Bidirectional Reflectance Characteristics," IEEE Transactions on Geoscience and Remote Sensing, 54(4), 2304-2313 (2016). 